\title{
SPECTROGRAPHIC OBSERVATIONS OF PECULIAR STARS. III*
}

\author{
P. Swings and O. Struve
}

\section{ABSTRACT}

This paper describes recent changes in the spectra of AX Per, Z And, AG Peg, and R Aqr. There is also a description of recent spectrograms of the unusually red bright-line star MWC 349 .

Our understanding of the physical, dynamical, and geometrical conditions prevailing in peculiar objects which combine bright lines of high excitation and late-type spectra will eventually be based on the spectroscopic variations in these stars. CI Cygni is the only complex object known which shows forbidden lines of high excitation which do not seem to have varied appreciably in recent years, although the late-type companion is variable. As far as the bright lines of high excitation are concerned, our spectrograms of CI Cygni since September, I939, do not differ from the ones taken by Merrill in I93 I and I932. ${ }^{\mathrm{T}}$ The present paper is concerned with the description and discussion of recent spectral changes in AX Persei, Z Andromedae, AG Pegasi, and R Aquarii. It also provides some additional information on the peculiar bright-line star MWC 349 .

$A X$ Persei.-This object, which in 1939 was similar to CI Cygni to such an extent that one could hardly distinguish their spectra, has suffered conspicuous variations. The I939 spectrum corresponded to a much higher ionization than that of I93I-I932, when it was described by Merrill. ${ }^{x}$ But on our spectrograms of January, I94I, the forbidden lines of $\left[F e\right.$ VII], which were extremely intense in I939, ${ }^{2}$ had disappeared, and other changes had also occurred. ${ }^{3}$ Now our spectrograms of August 2 and 8, I94I, have reversed the situation, the star having recovered a bright-line spectrum very similar to that of September, I939. [ $F e$ VII], practically absent in January, I94I, had recovered in August, I94I, its intensity of I939. A comparison of line intensities in the region $\lambda \lambda_{3869-6560}$ on January 5 , I94I, and August 8, I94I, is given in Table I; intermediate intensities are observed on a spectrogram secured on May 30, I94I. It is apparent that the general trend after January, I94I, has been toward an increase in excitation, which had reached a minimum around January, r94I. This increase is evidenced by the following changes in the intensities relative to $\mathrm{He}$ II and $H:(a)$ the large increase of $[F e$ VII] and $[N e \mathrm{v}]$; and $(b)$ the decrease in intensity of $H e \mathrm{I}, N$ III, $C \mathrm{III},[O \mathrm{III}]$, and [Ne III]. It should be noticed that the $H e$ I triplets have been reduced less than the singlets (compare, for example, $\lambda 4388$ and $\lambda 447 \mathrm{I}$ ).

$Z$ Andromedae.-After its I939 outburst to a mean maximum magnitude of 7.9 , $\mathrm{Z}$ Andromedae declined to a mean minimum brightness of 9.6 in 1940 and then increased again in I94I, reaching magnitude 8.7 in August, I94I. ${ }^{4}$ We noticed this recent outburst at the 82 -inch telescope on July 25 and August 6. Our spectrograms reveal very striking changes in this binary since August, I940, and even since January 5, I94I.

With regard to line intensities and structures, the evolution of the emission lines may be summarized as follows: (a) Increases in the following intensity ratios: $F e$ II/ $N$ III, $C$ III, $C$ IV; $O$ III fl./[Ne v]; $H e$ I/[Ne III]; $F e$ II/He I; $F e$ II/[O III $; S i$ II/Continuum; $M g$ II/Continuum. (b) No variation in the structure of the "nuclear" features in the

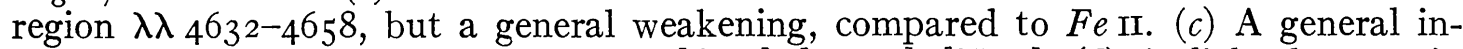
tensity decrease of the forbidden lines $[O \mathrm{III}],[N e \mathrm{III}],[N e \mathrm{v}] .(d)$ A slight decrease in

* Contributions from the McDonald Observatory, University of Texas, No. 42.

\footnotetext{
I $A$ p. J., 77, 44, I933.

${ }^{2} A p . J .$, 9 1, 607, r940.

3 Ibid., 94, 298, I94I.
}

${ }_{4}$ Harvard Announcement Card, Nos. 595 and 598; Leon Campbell, Pop. Astr., 49, 446, I94I. 
the ratio of the auroral to the nebular transitions of $[O \mathrm{III}]$ since I940 (no appreciab]e change since January, I94I). (e) A very strong Balmer continuum in emission.

The measured lines are shown in Table 2; the wave lengths have been corrected to the sun but have not been corrected for the motion of the star. For the identifications extensive use was made of our new table of wave lengths in a Cygni. ${ }^{5}$ In the region be-

TABLE 1

COMPARISON OF THE LINE INTENSITIES IN AX PERSEI

ON JANUARY 5, I94I, AND AUGUST 8, I94I

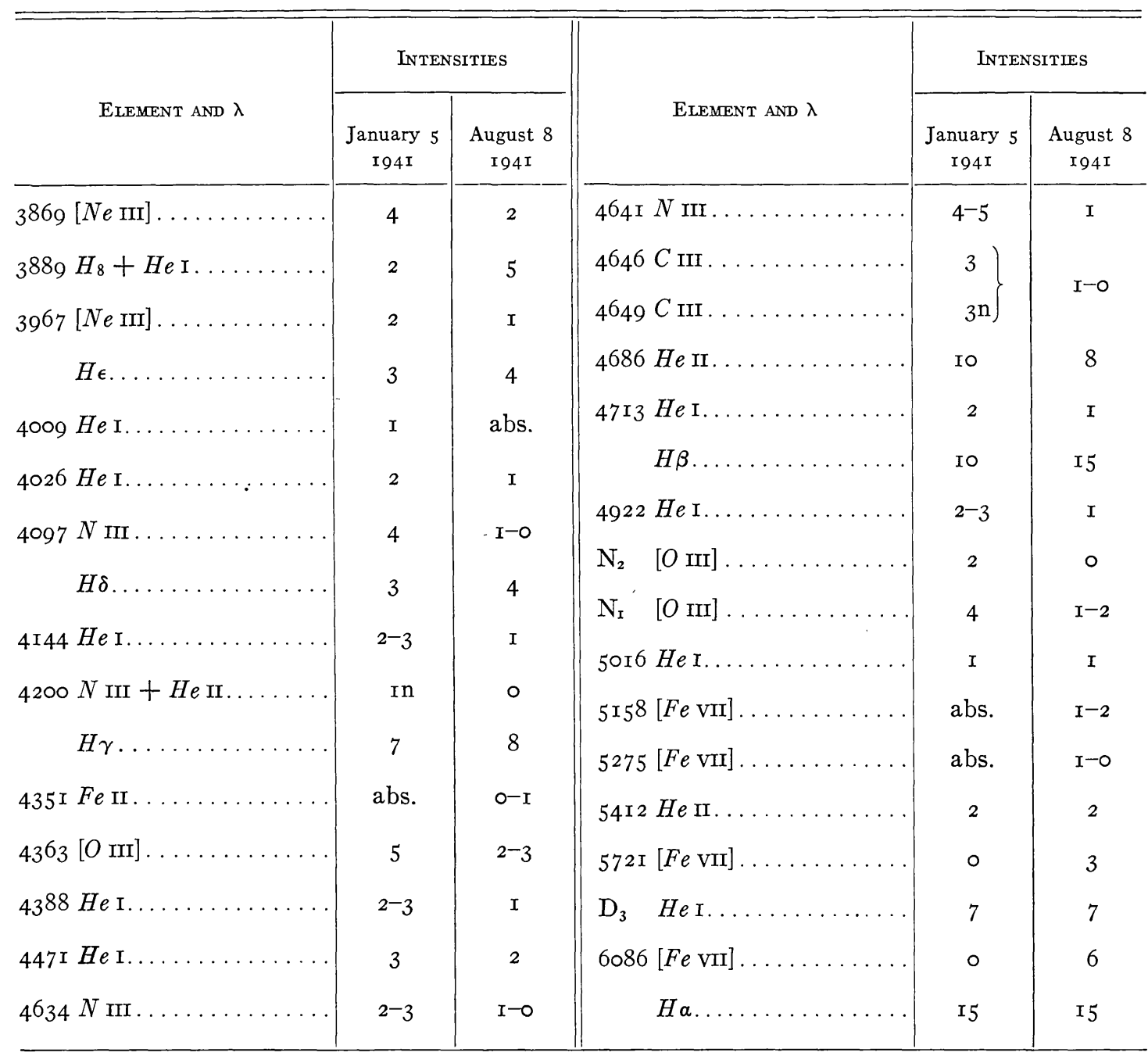

tween the Balmer limit and $\lambda 3445$, the very strong continuous hydrogen emission is interrupted only by the absorption lines $2 \mathrm{p}{ }^{3} \mathrm{P}^{\circ}-8$, 9, Iod ${ }^{3} \mathrm{D}$ of $H e \mathrm{I}\left(\lambda \lambda{ }_{36} 634,3587\right.$, and 3554); no trace is observed of $\mathrm{He}_{\mathrm{I}} 36 \mathrm{I} 3$, which means that the dilution effect is not important. This latter result is in agreement with the observations of a violet absorption component of the lines $2 \mathrm{p}{ }^{3} \mathrm{P}^{\circ}-5,6,7 \mathrm{~d}$ ' $\mathrm{D}$. He I 3634 cuts deeply into the Balmer continuum. The continuous spacings between the higher members of the Balmer

$5 A$ p. J., 94, 344, I94I. According to the variable-star observers of the Milwaukee Astronomical Society, the apparent visual magnitude of $Z$ And was 8.8 at the time of our spectroscopic observations. 
TABLE 2

SPECTRUM OF Z ANDROMEDAE (JULY-AUGUST, I94I)

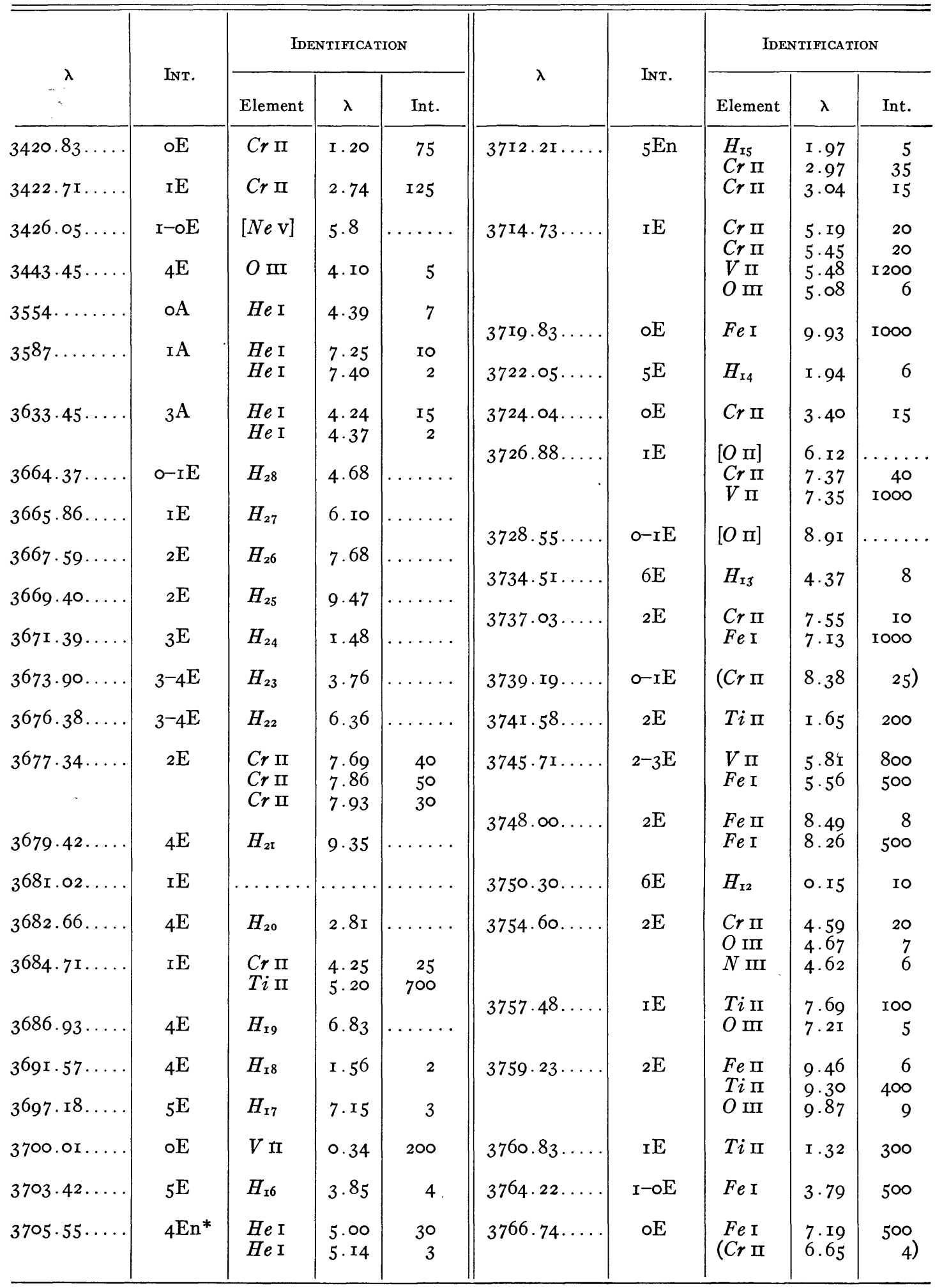

* Faint violet absorption. 
TABLE 2-Continued

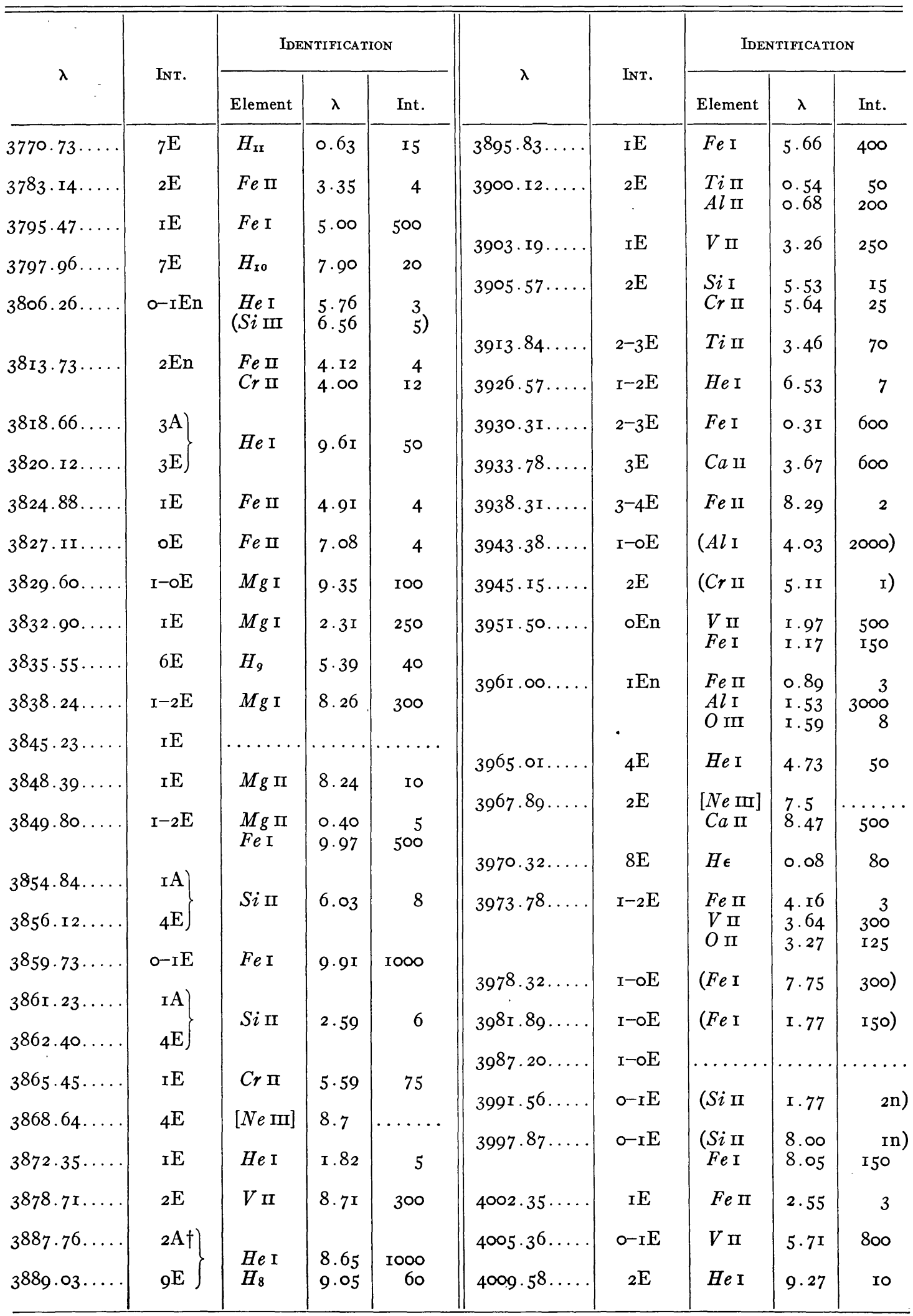

† The absorption component is due to $H e \mathrm{r}$ only. 
TABLE 2-Continued

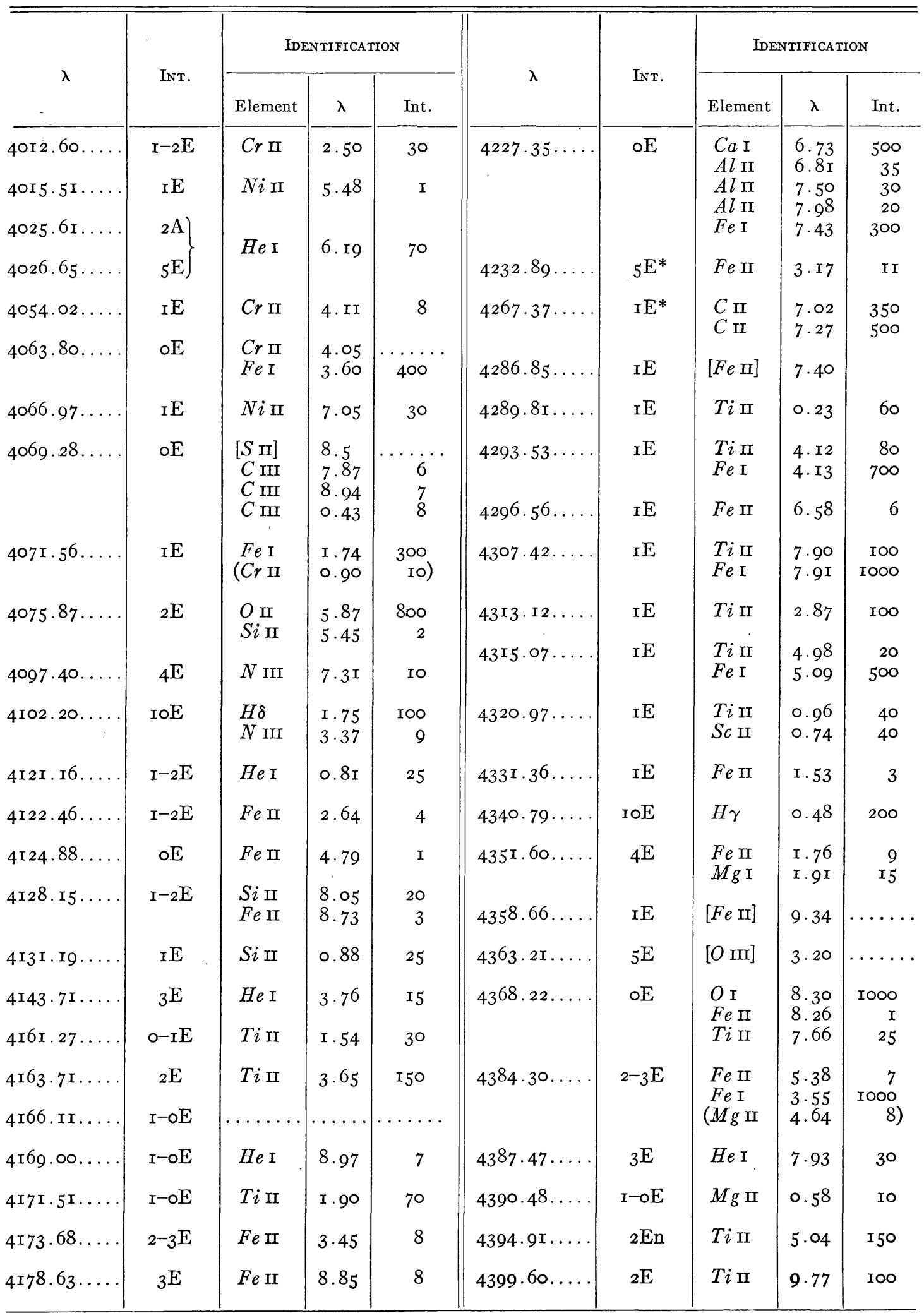


TABLE 2-Continued

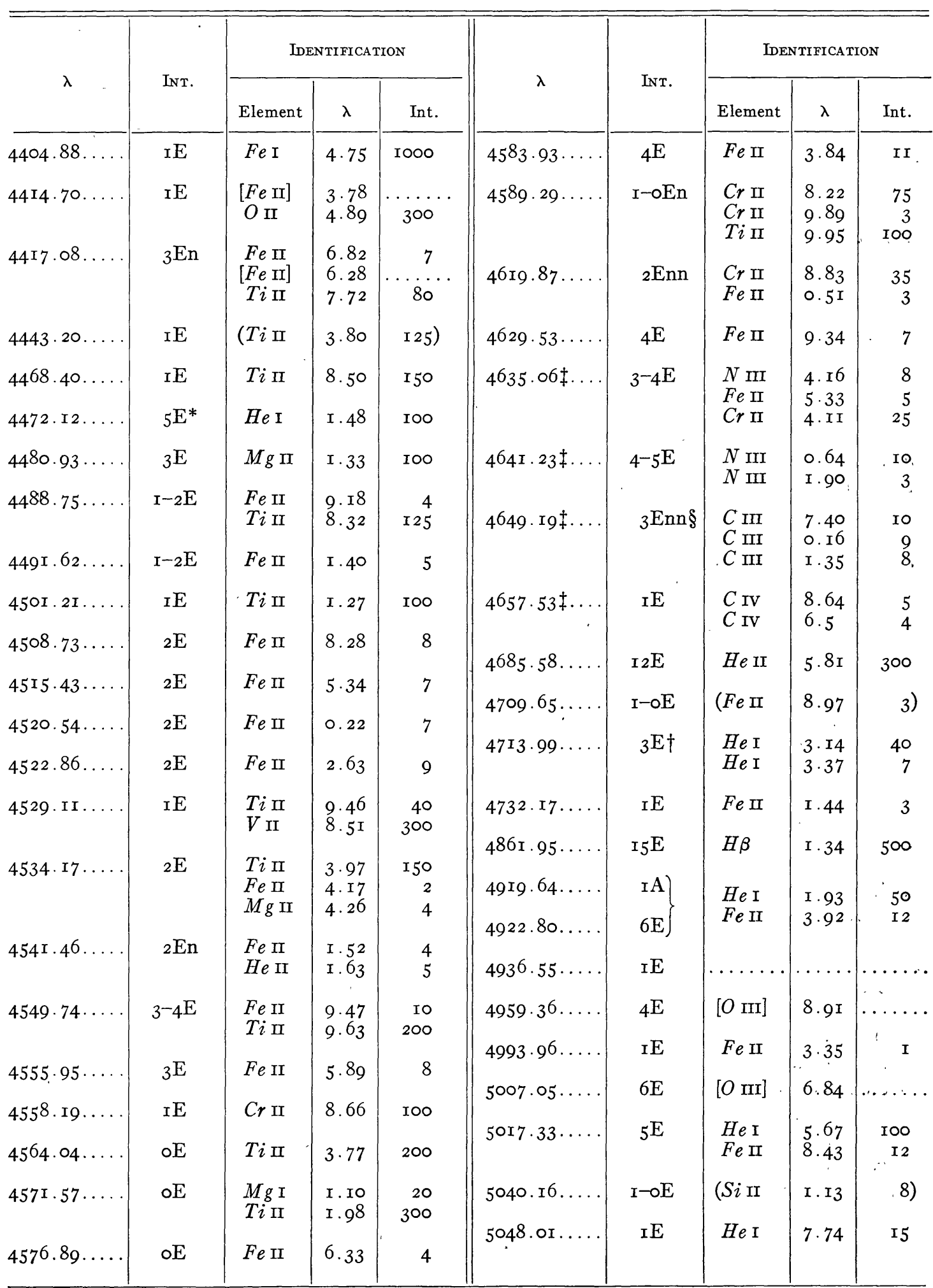
$\ddagger$
r940.

‡These "nuclear" features have a violet absorption component, and their general structure has not changed since August,

8 Extends from $\lambda_{4}{ }_{4} 6.3$ to $\lambda_{4} 65$ r.9. 
TABLE 2-Continued

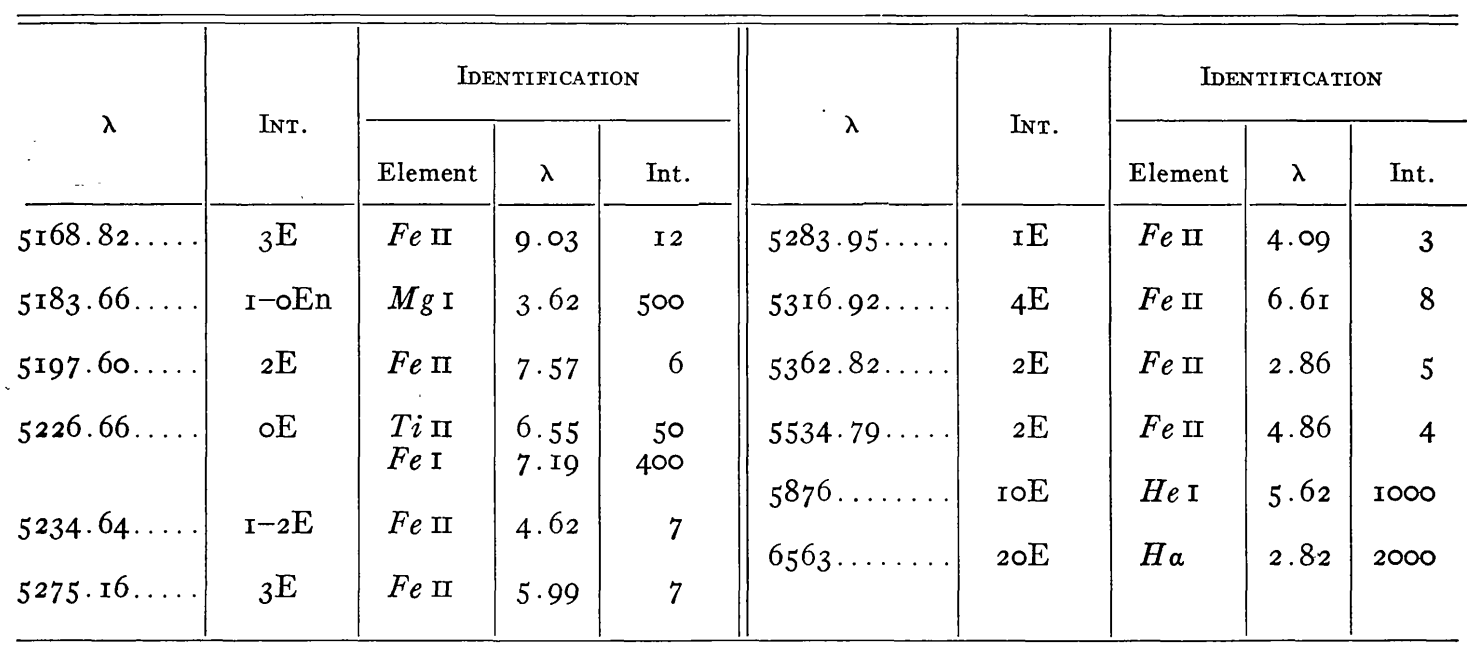

series are faint; this shows that the continuous spectrum at $\lambda_{36} \mathrm{I}_{3}$ is practically all due to the Balmer continuum. Hence, this continuum is absorbed by overlying $\mathrm{He} \mathrm{I}$ atoms.

The radial velocities on August 6, I94I, have the following values:

$H:$

He I:

Si II:

Metallic ions: $\quad V_{\mathrm{em}}$ of $\mathrm{Fe}$ II: $-2 ; \mathrm{Ti} \mathrm{II}:-\mathrm{r} 3 ; \mathrm{Cr} \mathrm{II}:-\mathrm{II} ; \mathrm{Mg} \mathrm{II}:-27 ; \mathrm{Ca} \mathrm{II}:+8$

High excitation

features:

$$
\left.V_{\mathrm{em}}=+9 \text { (against }+6 \text { in } 1940\right)
$$

Nuclear fea-

tures:

$$
\text { No variation in either } V_{\mathrm{em}} \text { or } V_{\mathrm{abs}} \text { since } \mathrm{r} 940
$$

On the whole, we may say that all the emission lines not accompanied by violet absorptions have about the same radial velocity; that the velocity of expansion of $\mathrm{He} \mathrm{I}$ is essentially the same as in I940; that $H e$ I and $S i$ II have practically the same ejection velocities; and that the "nucleus" and the "forbidden nebular lines" have, as a whole, simply become fainter in the course of I94I.

Whatever may be the true origin of the "nuclear" features-either an exciting WolfRayet nucleus or deep layers in the nebula-their intensity decrease, which was simultaneous and similar to that of the forbidden nebular lines, is easily understood. The other spectroscopic variations observed in I94I are presumably due to the fact that more abundant matter was ejected with practically the same velocity as last year. Part of the emissions and violet absorptions (excluding the "nuclear" features) arise in the new 
shell, which is still fairly close to the photospheric surface. The opacity of the new shell reduces the excitation in the nebula.

This general picture is crude and qualitative; but it is hoped that additional observations may provide data for a more or less quantitative treatment.

$A G$ Pegasi $\left(B D+I I^{\circ} 4673\right)$. - The general trend toward higher excitation has continued. ${ }^{6}$ Comparing our I94I spectrograms ${ }^{7}$ with those of $1939-$ I940, it is apparent (I) that $S i$ I 3905 has disappeared; (2) that $C a$ II and $F e$ II have decreased in intensity; and (3) that $\mathrm{He}$ II, $N$ III, and $S i$ IV have increased appreciably.

$R$ Aquarii.-Spectrograms of this object secured on July 30 and August I2, I94I, when the late-type companion was near minimum, ${ }^{8}$ reveal that this peculiar star has recovered its $[F e$ III $]$ stage. ${ }^{6}$ The $[F e$ III $]$ lines were observed by Merrill from I9I9 to I926, inclusive, when the nebular spectrum was strong, as a whole. On a I924 plate, $\lambda_{46} 8$ was "probably accompanied by $\lambda 470$ r and $\lambda$ 4733." In I939 there was no trace of [Fe III]. The emission lines observed on our spectrograms of August, I94I, are:

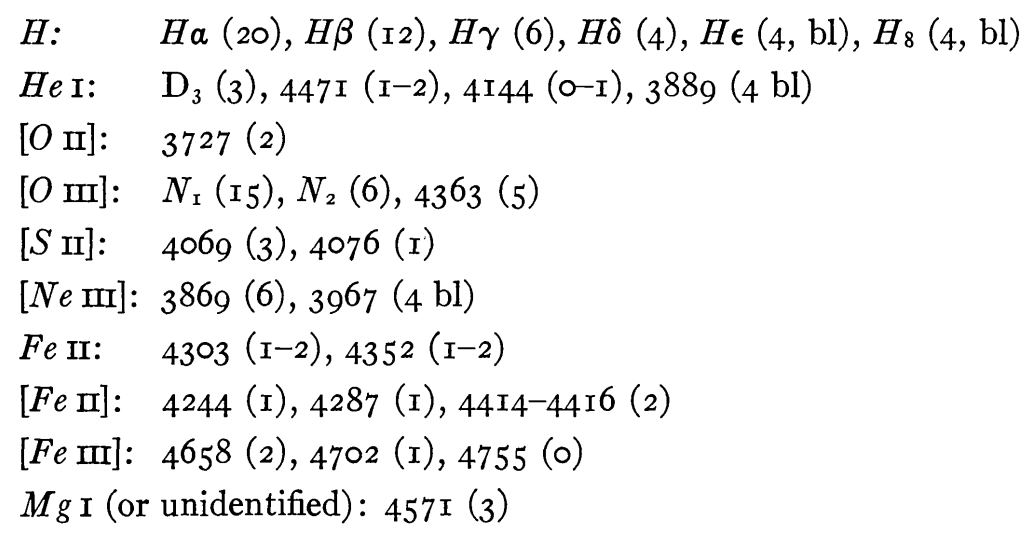

The presence of the transitions of nebular type of $[O \mathrm{II}]$ is interesting, since they have an extremely low transition probability; they must undoubtedly arise in a nebular region of extremely low density.

$M W C 349 .{ }^{9-}$ The line emission in this star has been described by Merrill, Humason, and Burwell as follows:: "In addition to the hydrogen lines, the bright nebular line at $\lambda_{4658}$ is well marked and the companion line $\lambda 470 \mathrm{O}$ is visible. Bright lines of neutral helium are also present and possibly $\lambda 4583$ of ionized iron."

MWC 349 bears a striking spectral analogy to MWC $\mathrm{I} 7^{\text {II }}$ and should also be compared to the star of slightly higher excitation, RY Scuti. ${ }^{12}$ It is located in a dark region of the Milky Way and is reddened to a considerable extent; the total absorption is probably of the order of Io mag.

${ }^{6}$ This was also observed by Dr. P. W. Merrill (communication at the Yerkes Observatory Symposium on Stellar Spectra, September ro-r2, I94I).

${ }^{7}$ According to the Milwaukee observers, the magnitude of AG Peg was 7.7 at the time of our spectroscopic observations in July and August, r94I.

${ }^{8}$ According to the Milwaukee observers, the magnitude of R Aqr was r r.o at the time of our spectroscopic observations.

${ }_{9} a(1900): 20^{\mathrm{h}} 29^{\mathrm{m}} \cdot 2 ; \delta(\mathrm{r} 900):+40^{\circ} \mathrm{I} 9^{\prime} ; 22^{\mathrm{s}}$ preceding and $3^{\prime}$ north of $\mathrm{BD}+40^{\circ} 4226$. Magnitude given by Merrill in I932: 13.2 ; the star was fainter than the fourteenth magnitude when observed on July $3 \mathrm{I}$ and August I, r94I. Galactic co-ordinates: $l=47^{\circ}, b=0^{\circ}$.

го $A$ p. J., 76, г56, 1932, star MW 203.

II $A$ p. J., 93, 349, I94 r. $\quad$ ×2 $A p . J .$, 9 r, 58r, r940. 
The lines observed on our spectrograms are:

$H: \quad \cdot H a(20), H \beta(3), H \gamma(\mathrm{I})$

$\mathrm{He} \mathrm{I}: \quad D_{3}(7)$

[O I]: $6300(2)$

[N II]: 5755 (5)

Fe II: $\quad{ }_{5169}$ (I), 53 I 7 (I-O)

[Fe III]: 5270 (3), 5010 (2), 4658 (I), 470I (o)

The reddening is especially noticeable in the $[F e \mathrm{III}]$ lines. In unreddened stars, $\lambda_{46} 68$, which is the leading component of the $5 \mathrm{D}-{ }^{3} \mathrm{~F}$ multiplet, is always at least as strong as $\lambda_{5270}$, which is the strongest $5 \mathrm{D}-{ }^{3} \mathrm{P}$ transition, and $\lambda{ }_{5010}$ is always weaker than $\lambda{ }_{4658}$. But in MWC $349, \lambda_{5270}$ and $\lambda_{5010}$ are appreciably more intense than $\lambda 4658$. This gives conclusive evidence in favor of a considerable color excess (of the order of 2 mag.) and consequently of a tremendous general absorption. The general intensity distribution in the continuous spectrum is similar to a late $\mathbf{M}$ star, but no latetype absorption feature is apparent. By comparison with RY Scuti we should expect the spectral type to be early $B$.

In RY Scuti the $[F e \mathrm{III}]$ lines are also strong, but $H e \mathrm{II}$ is present and $\mathrm{Fe}$ II is absent; in $\mathrm{MWC} \mathrm{I}_{7}$ both $\mathrm{Fe}$ II and [ $\mathrm{Fe}$ III] are present, and $\mathrm{He}$ II is absent.

MCDonald ObSERVATORY

September I94I 